\title{
Proper Care of Allergic Asthma before Hospitalization
}

\section{Mohammad Amin Valizade Hasanloei ${ }^{1}$ and Seyyed Shamsadin Athari ${ }^{2 *}$}

${ }^{1}$ Anesthesiologist-Fellowship of Intensive Care Medicine, Urmia University of Medical Science, Iran

${ }^{2}$ Young Researchers and Elite Club, Tabriz Branch, Islamic Azad University, Iran

Allergic asthma is one of the most common chronic diseases of childhood and multifactorial disease that influenced by genetic and environmental factors with intermittent symptoms of cough, dyspnea, wheezing and chest pain. The increase in allergic diseases was reconciled with the hygiene hypothesis. When hygiene is in high quality and there are low infections, allergic problems are increasing. Strongrelationship between allergic diseases and HLA antigens has beendemonstrated. That is characterized by bronchial hyper-responsiveness and variable degrees of airways obstruction. Asthma is a cause of substantial mortality and morbidity and has a considerable economic impact. Physicians, who care of people with allergic asthma, have some urgent and intensive care unit (ICU) hospitalization of these [1,2].

Immunological and inflammatory process is the underlying cause of asthma attacks that are predominately related to tissue reactions. The immunological and inflammatory stages are the target steps for the controller. Allergic asthma is also associated with high direct and indirect health costs, especially related with diagnosis and treatment. Usual asthma drugs for example Inhaled corticosteroids can also have systemic side effects, including arterial hypertension, diabetes, and obesity. Therefore, avoidance of specific allergens and other precipitating factors is helpful in the management of asthmaand prevention of ICU hospitalization [1-3].

Mortality among patients hospitalized for asthma exacerbations accounts for notable number of all deaths from asthma. Management of asthma exacerbations before hospitalization and during transportation to the emergency department could be reducing the overall risk of death and in eliminating race disparities in asthma deaths.

\section{References}

1. Seyyed Shamsadin Athari (2013) Traditional Medicine for Asthma. Advances in Biological Research 7: 112-113.

2. Seyyed Shamsadin Athari (2013) Immune Response Shifting of Asthma in Aging. Middle-East Journal of Scientific Research 13: 489-498.

3. Seyyed Shamsadin Athari (2013) Best Treatment for Allergic Asthma with Traditional Herbal Medicine: A Brief Report. American-Eurasian J Agric and Environ Sci 13: 291-292.
*Corresponding author: Seyyed Shamsadin Athari, Young Researchers and Elite Club, Tabriz Branch, Islamic Azad University, Tabriz, Iran, Tel: +989143044606; E-mail: SS.Athari@gmail.com

Received January 04, 2014; Accepted January 30, 2014; Published February 05, 2014

Citation: Hasanloei MAV, Athari SS (2014) Proper Care of Allergic Asthma before Hospitalization. J Allergy Ther 5: 161. doi:10.4172/2155-6121.1000161

Copyright: @ 2014 Hasanloei MAV, et al. This is an open-access article distributed under the terms of the Creative Commons Attribution License, which permits unrestricted use, distribution, and reproduction in any medium, provided the original author and source are credited. 